\title{
L'HISTOIRE DE LA GÉOGRAPHIE OBJETS, ENJEUX ET PERSPECTIVES
}

L'histoire de la géographie, tout comme la géographie elle-même, s'élabore à la confluence de problématiques multiples, porteuses d'interrogations pressantes sur ses objets, démarches et conditions de pertinence scientifique et sociale.

Étant exclu d'en proposer ici ne serait-ce qu'une ébauche d'inventaire, on s'attachera plutôt à en organiser le survol à l'aide de trois thèmes parmi les plus éclairants ${ }^{1}$.

\section{I. - LES OBJETS DE L'HISTOIRE DE LA GEOGRAPHIE}

Cette première question portant sur l'étendue et la nature du champ d'étude renvoie immédiatement à une autre, celle de la "géographicité " entendue comme domaine d'extension du savoir et/ou des pratiques géographiques. De cette définition préliminaire dépendent non seulement la spécificité (et le volume) du corpus de phénomènes qui seront pris en considération, mais également les méthodes et démarches explicatives mises en cuvre.

La question est donc capitale, même si trop souvent on croit pouvoir l'éluder soit par le biais de la récuperation implicite de la conception de la géographie (localisée et datée) propre au chercheur, soit par la désignation de moments ou de personnages fondateurs dont le choix est souvent passablement arbitraire.

1. Pour des approches complémentaires ou différentes de la nôtre, voir, par exemple : René Clozier, Histoire de la géographie, Paris, P.U.F., 1967; Preston James, Geoffrey Martin, All Possible Worlds, New York, John Wiley and Sons, 1981 ; Ronald Johnston, "On Geography and the History of Geography", History of Geography Newsletter, 3, 1983, p. 1-7. Certaines questions seulement effleurées ici sont développées dans : Daniel DORY, "A propos de quelques problèmes théoriques de l'histoire de la gégraphie ", Union géographique internationale, Conférence régionale de Barcelone, 1986.

Revue de synthèse: IV S. Nos 3-4, juil.-déc. 1988. 
Or, la sélection et la mise en ordre des matériaux hétérogènes qui constituent les sources de l'histoire de la géographie sont directement tributaires de cette élucidation préliminaire. Ainsi, poser, comme nous le faisons, que le champ de l'histoire de la géographie s'inaugure avec les premières représentations liées à la transformation des milieux naturels en territoires, au cours des processus sociaux de production et de reproduction des biens et des personnes, a des conséquences multiples pour l'entreprise historienne. La plus significative d'entre elles étant de faire émerger un corpus de données que l'on peut regrouper schématiquement dans les cinq catégories suivantes:

a) Les représentations archaïques et ethnogéographiques reflétant les premiers moments des rapports entre Sociétés et Nature (au moyen, notamment, de dispositifs technologiques à efficacité paysagique limitée). Leur diversité et leur richesse commencent seulement à être mesurées tant dans les sociétés traditionnelles qu'au sein de systèmes de pensée plus élaborés, sacrés ou profanes ${ }^{2}$.

b) Les images successives de la Terre depuis les voyages antiques jusqu'à la télédétection contemporaine, en passant par les explorations diverses (et non seulement européennes) ${ }^{3}$.

c) Les représentations changeantes (cartographiques surtout, mais non exclusivement) des mondes connus et/ou imaginés ${ }^{4}$.

d) Les transformations techniques et sociales ayant permis une appropriation croissante des ressources terrestres donnant lieu à des paysages de plus en plus façonnés par l'action anthropique 5 .

e) Les avatars de la pensée géographique, à la fois reflet de pratiques sociales concrètes et réflexion en quête d'une scientificité et d'une opérationnalité croissantes ${ }^{6}$.

Reste alors à l'analyse historique d'élucider les modes d'articulation variables de ces différents ordres de phénomènes suivant l'époque envisagée et la problématique de la recherche.

2. Parmi les travaux sur cette question: Peter HUBER, «Angoor Floods : Reflections on Ethnogeography and Mental Maps ", The Geographical Review, vol. 69, 2, 1979, p. 127139; Tetsuya HisataKe, "Indigenous Maps, Cosmology, and Spatial Recognition of the North American Indian : With Special Reference to the Ojibway Around Lake Superior "w, in Hideki NozAwa, ed., Cosmology Epistemology and the History of Geography, Kyushu University, Fukuoka, 1986, p. 1-25; Hong-Key YoON, "The Nature and Origin of Chinese Geomancy ", Eratosthene-Sphragide, 1, 1986, p. 88-102.

3. Pour un premier survol de la matière : Hubert Deschamps, Histoire des explorations, Paris, P.U.F., 1969.

4. Philippe Pinchemel, "Géographie et cartographie. Réflexions historiques et épistémologiques", Bulletin de l'Association des géographes français, 463, 1979, p. 239-247.

5. Problématique abordée par Geneviève et Philippe PinCHEMEL, "Réflexions sur l'histoire de la géographie. Histoires de la géographie, histoire des géographies », C.T.H.S., Bulletin de la Section de géographie, t. LXXXIV, 1981, p. 221-231.

6. Paul Claval, La Pensée géographique, Paris, SEDES, 1972. 
On le voit, la conception de la "géographicité " qui sous-tend notre démarche historique est extrêmement englobante. Ce n'est pourtant qu'à cette condition que l'on peut éviter les pièges complémentaires d'anachronisme et de légitimation rétrospective de l'état actuel de la discipline. En d'autres termes ceci permet d'envisager la géographie contemporaine comme un moment explicable et transitoire d'organisation des savoirs portant sur la Terre et son aménagement, et non pas comme l'aboutissement nécessaire de moments antérieurs conçus dans une perspective vaguement téléologique.

C'est aussi grâce à l'horizon théorique que constitue cet ensemble complexe de questions qu'il devient possible de donner cohérence aux études particulières indispensables à l'avancement de la recherche. En effet, à défaut d'une perspective globale sur le domaine étudié, le risque est grand de n'assister, pour longtemps encore, qu'à l'accumulation d'entreprises monographiques, souvent précieuses mais pratiquement incomparables, ou encore de voir confondre chronologie et histoire par défaut $d$ 'hypothèses directrices.

Dernier point enfin, pour lequel la définition de l'objet de l'histoire de la géographie s'avère crucial : l'élaboration de stratégies d'investigation portant prioritairement l'attention vers les périodes et conjonctures les plus révélatrices.

Un certain nombre d'entre elles ont d'ailleurs fait l'objet de travaux qui en ont montré l'importance. Citons, à titre d'exemple, les études concernant l'institutionnalisation de la géographie en Europe occidentale dans la seconde moitié du $\mathrm{XIX}^{e}$ siècle$^{7}$, l'épanouissement et la différenciation des écoles géographiques nationales ${ }^{8}$, l'évolution de la pensée et des idées géographiques, etc. Par contre, force est de constater un certain retard dans la prise en compte systematique de thèmes tels que l'ethnogéographie ${ }^{10}$, les rapports entre formations

7. Cr. Horacio CAPEL, "Institutionalization of Geography and Strategies of Change ", in David R. STODDART, ed., Geognaphy, Ideology and Social Concern, Oxford, Basil Blackwell, 1981, p. 37-60. Cf. également Vincent Berdoulay, La Formation de l'Ecole française de géographie (1870-1914), Paris, CTHS/Bibliotheque nationale, 1981.

8. La revue polonaise Organon, 14,1980 , a réuni un ensemble de contributions sur $\infty$ thème.

9. Parmi les nombreuses publications consacrées à cette question, cf. P. JAMES, G. MARTIN, op. cit. supra n. 1, P. Claval, op. cit. supra n. 6 et D. R. STOddarT, op. cit. supra n. 7. Pour un aperçu sur les débats actuels en géographie, on consultera utilement Milton E. HARVEY, Brian P. Holly, eds, Themes in Geographic Thought, Londres, Croom Helm, 1983.

10. Les recherches, encore embryonnaires dans le domaine de l'ethnogéographie, auront à s'appuyer, en un premier temps, tout au moins, sur les travaux anthropologiques concernant les « ethnosciences ", en procédant à l'étude détaillée des aspects géographiques des mythes, cosmologies et représentations populaires. Pour une approche d'ensemble du 
économiques et sociales, techniques et pensée géographique, pour l'étude desquels les géographies coloniales forment un laboratoire hors pair ${ }^{11}$, etc. L'explication de cet inégal développement de l'histoire de la géographie étant très largement à chercher dans les circonstances et les enjeux entourant sa pratique.

\section{II. - UNE hISTOIRE AUX ENJEUX MULTIPLES}

La recherche en histoire de la géographie est essentiellement menée par des géographes et s'adresse, jusqu'à présent, surtout à d'autres géographes. Il s'agit là d'un fait important permettant d'en saisir certains enjeux théoriques (et parfois pratiques). En effet, ces circonstances particulières font que, quelle que soit par ailleurs la rigueur méthodologique et critique des travaux entrepris, le géographe, analysant l'histoire de telle ou telle branche ou période de sa discipline, se trouve nécessairement devoir prendre position (implicitement ou explicitement) sur l'état actuel de la géographie et les modalités et conditions contemporaines de son exercice. Cette caractéristique - que l'histoire de la géographie partage avec celle de la plupart des sciences sociales - tenant dans une grande mesure à l'absence d'une communauté d'historiens spécialisés des sciences se consacrant à la géographie sans pourtant être eux-mêmes géographes, n'est pas à déplorer en tant que telle, à la condition d'en analyser les risques et potentialités avec soin.

Ceci n'est possible, en l'occurrence, qu'en prenant la mesure de deux spécificités majeures de la géographie elle-même, l'une structurelle, l'autre conjoncturelle. Tout d'abord, il convient d'insister sur le fait que si la géographie est une science sociale, elle n'est point seulement cela. De fait, ayant pour objet l'ensemble des conditions et conséquences des relations entre les sociétés et les milieux naturels, la géographie se situe à l'intersection de deux ordres de savoirs entre lesquels opère une coupure parmi les plus efficaces de celles qui partagent le champ scientifique contemporain. Cette gestion simultanée de ses versants sociologique et naturaliste étant pour la géographie à la fois source

versant naturaliste de la question, cf. Jacques BARRAU, « Des modes de connaissance de la nature ", La Pensée, 220, 1981, p. 60-68.

11. Cf., par ex. : Mohamed NACIRI, « Une géographie coloniale ", Hérodote, 13, 1979, p. 36-59; D. DORY, « Entre la découverte et la domination : Le Lobi (1800-1960). Éléments d'histoire de la géographie coloniale ", Bulletin de l'Association des géographes francais, 506,1984 , p. $373-382$. 
d'un prodigieux intérêt scientifique et de bon nombre de crises épistémologiques, voire existentielles.

La deuxième particularité, découlant d'ailleurs partiellement de la première, concerne la conjoncture actuelle de la géographie. Celle-ci se caractérise par un foisonnement d'hypothèses et d'orientations diverses, souvent d'un grand intérêt, mais dissimulant mal de profondes divergences (avouées ou non) quant à la nature, la structure et l'objet de la discipline.

Cette crise d'identité trouve pour une bonne part ses racines dans les deux ébranlements majeurs qu'a connus la géographie au cours du $\mathrm{XX}^{e}$ siècle. Le premier fut la conséquence d'une série de réponses peu satisfaisantes apportées aux questions mal posées par le déterminisme environnemental mécaniste du début du siècle.

Les diverses variantes de possibilisme ou d'indéterminisme qui s'affirmeront alors, en barrant la voie menant à une saisie des relations Sociétés-Nature en termes d'interaction dialectique, allaient conduire, d'une part au triomphe - éphémère mais dévastateur - des monographies littéraires idéographiques et incomparables et à la scission, durable, elle, entre une géographie physique louchant de plus en plus en direction des sciences naturelles "pures" et une géographie humaine engagée dans la quête alchimique désespérée de la pierre philosophale qui transformerait en "noyau dur " géographique l'amoncellement hétéroclite de méthodes, faits et théories à la mode puisés dans les chantiers des sciences sociales.

Le second bouleversement s'opéra au cours des années 1950-1960 sous la forme initiale d'une vague d'innovations méthodologiques et techniques (introduction de l'analyse mathématique, utilisation de l'informatique, modélisation, etc.). Cette "révolution quantitative " 12 revendiquait par ailleurs un projet nomothétique, saine réaction face à l'impressionnisme ambiant. Cependant, le contexte de cette " révolution " (issue des courants les plus technocratiques de la géographie américaine), l'indigence de la réflexion épistémologique l'accompagnant, ainsi que la récupération non critique des postulats les plus discutables de la vulgate libérale ${ }^{13}$, allaient conduire ce qui aurait pû être une rectification fructueuse dans l'impasse théorique et pratique d'une géométrie spatialiste aggravant encore l'écartèlement interne de la géographie et son

12. Parmi l'énorme littérature consacrée à ce sujet, cf. Ian BURTON, « The Quantitative Revolution and Theoretical Geography ", The Canadian Geographer, vol. 7, 4, 1963, p. 151162 ; et pour une brève mise au point plus récente, Hubert BEGUIN, "Analyse quantitative ", in Antoine S. BAILly, éd., Les Concepts de la géographie humaine, Paris, Masson, 1984, p. 163-171.

13. Cf., par ex. : Alain Rallet, "Les aventures de l'espace en économie politique", EspacesTemps, 26-27-28, 1984, p. 118-130. 
isolement dans la cité scientifique où son prestige intellectuel ne cessa de décroître.

Les tentatives actuelles de recentrer la géographie ${ }^{14}$ et d'en préciser les bases ${ }^{15}$ s'effectuent donc sur cette toile de fond, qui est en même temps celle de l'histoire de la géographie. On comprend dès lors aisément que, dans les débats actuels, cette histoire constitue elle-même un enjeu de taille; l'historien de la géographie se trouvant par conséquent dans la situation inconfortable de celui qui s'avance en terrain miné tout en portant des explosifs qu'il dispose çà et là, chemin faisant...

\section{III. - PERSPECTIVES ET OUVERTURES}

Les particularités de l'histoire de la géographie, dont il vient d'être question, ne doivent toutefois pas occulter l'importance de ses acquis et l'intérêt de ses débats pour l'ensemble de la communauté (disparate, il est vrai) des historiens des sciences.

Ainsi, par exemple, si la question des avatars du statut historique d'un savoir relevant à la fois des sciences de la nature et de celles de la société s'est posée et se pose encore de manière particulièrement dramatique en géographie (car c'est l'existence même de la discipline qui s'y joue), d'autres sciences de l'Homme sont également porteuses d'interrogations similaires. Pour ne prendre que deux cas particulièrement significatifs, mentionnons l'anthropologie et la psychologie. L'histoire de la géographie en véhiculant (et en résolvant partiellement) un certain nombre de problèmes généraux de l'histoire des sciences (clivages disciplinaires, processus de "disciplination " et de «coagulation " institutionnelles des sciences...) possède donc une portée cognitive dépassant le seul domaine de la géographie.

A certains égards on peut même avancer que par la nature des problèmes qu'elle pose, l'étendue de son champ (dans le temps mais aussi à l'échelle mondiale qui est nécessairement la sienne ${ }^{16}$ ), la

\footnotetext{
14. P. PINCHEMEL, " De la géographie éclatée à une géographie recentrée ", Tidjdschrift Voor Econ. en Soc. Geografie, vol. 73, 6, 1982, p. 362-369.

15. D. DORY, « La géographie fondamentale: de l'exigence au projet ", contribution au colloque "Géographie : Etat des lieux ", organisé par EspacesTemps et les Cahiers de géographie du Québec, à paraitre en 1988.

16. La diversité des géographies passées et présentes est de mieux en mieux perçue gráce à la publication d'ouvrages aux visées comparatives tels que : Orlando PENA, AndréLouis Sanguin, El Mundo De Los Geografos, Barcelone, Oikos-Tau, 1984; Ronald
} 
coordination croissante de ses recherches ${ }^{17}$ et l'affinement progressif de ses hypothèses, l'histoire de la géographie est en mesure de devenir un révélateur privilégié d'enjeux parfois moins visibles mais non moins présents dans d'autres domaines scientifiques. Retraçant tour à tour des appropriations territoriales et intellectuelles, des découvertes fondamentales, des affrontements méthodologiques et des déchirements internes, l'histoire de la géographie se trouve nécessairement mise en demeure d'élaborer une réflexion sur l'ensemble des problématiques de l'histoire des sciences. Parmi celles-ci, la mise en question des origines et des modalités d'approfondissement du clivage entre sciences naturelles et sociales, dont la portée épistémologique est immense, occupe une place privilégiée. C'est que, pour se développer et s'affirmer, la géographie se doit aussi de dater la genèse des forces centrifuges qui agissent en son sein, condition première de leur analyse d'abord et de leur éventuelle neutralisation ensuite.

Au terme de ce bref survol de l'histoire de la géographie telle qu'elle s'élabore aujourd'hui, quelques spécificités retiennent l'attention.

Tout d'abord c'est l'étroite liaison entre les débats traversant la géographie contemporaine et les problématiques mises en œuvre par les historiens de la géographie. Ceci du fait que, l'entreprise historienne, quel que soit par ailleurs son souci méticuleux d'exactitude, se trouve, par le choix de ses objets et la nature des interrogations auxquelles elle les soumet, engagée dans la légitimation ou la récusation, implicite ou explicite, des pratiques géographiques actuelles.

Mais ce n'est pas tout. Au-delà du souci d'exhiber des ancêtres prestigieux et de célébrer les exploits du passé qui caractérisent tout domaine au statut épistémologique fragile, l'histoire de la géographie dévoile les modalités suivant lesquelles un ensemble de pratiques, techniques et théoriques, dont les origines se situent à l'aube de l'anthropogenèse, a fini par se constituer en discipline scientifique à l'identité aujourd'hui problématique.

Sa dualité intrinsèque apparaît alors clairement. A la fois entreprise

\footnotetext{
Johnston, Paul Claval, Geography since the Second World War, Londres, Croom Helm, 1984. Voir également l'article de David Hooson, "Geography East and West ", Geojournal, vol. 14, 2, 1987, p. 203-209.

17. En France, l'équipe de l'U.A. 04.914 Université Paris I-C.N.R.S. «Epistémologie et histoire de la géographie ", dirigée par M. le prof. P. Pinchemel constitue la base institutionnelle de l'histoire de la géographie. Au plan mondial, un groupe de travail se consacre à l'histoire de la pensée géographique dans le cadre de l'Union géographique internationale, et ce, depuis 1968. Voir à ce sujet: P. PinCHEMEL, "La Commission d'histoire de la pensée géographique de l'Union géographique internationale ", in Jozef BABICZ, éd., Études d'histoire de la géographie et de la cartographie, Wroclaw, Académie polonaise des sciences, 1973, p. 59-66.
} 
indispensable pour approfondir la réflexion sur les fondements de la science géographique, cette histoire porte aussi témoignage de l'immense effort humain d'appropriation de la Terre. Ces deux composantes n'étant aucunement exclusives, l'avenir de l'histoire de la géographie sera, au moins partiellement, le produit de leur conjugaison.

Daniel DoRY, C.N.R.S., Université de Paris I. 